\title{
Protective function of Bu Shen Huo Xue formula on the immunity of B6AF1 mice with experimental autoimmune premature ovarian failure
}

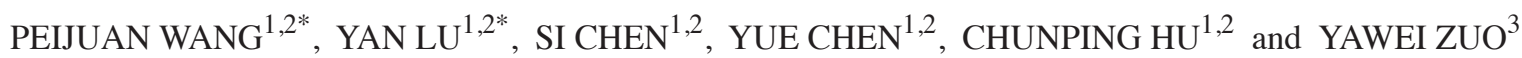 \\ ${ }^{1}$ Department of Obstetrics and Gynecology, Affiliated Hospital of Integrated Traditional Chinese and Western Medicine, \\ Nanjing University of Chinese Medicine; ${ }^{2}$ Department of Obstetrics and Gynecology, Jiangsu Province Academy of \\ Traditional Chinese Medicine, Nanjing, Jiangsu 210028; ${ }^{3}$ Infirmary, 8724 Forces Health Teams, \\ Wuxi, Jiangsu 214000, P.R. China
}

Received September 14, 2016; Accepted July 27, 2017

DOI: 10.3892/etm.2018.5804

\begin{abstract}
Autoimmune abnormality is one of the main causes of premature ovarian failure (POF). Bu Shen Huo Xue formula (BSHXF), a traditional Chinese medicine formula, has been clinically used for the treatment of patients with POF in China. Regulatory T cells (Tregs) are important in the pathogenesis of autoimmune POF. The aim of the present study was to evaluate the immunoprotective effects of BSHXF on POF and the underlying mechanisms. An experimental autoimmune POF model was induced in B6AF1 mice with zona pellucida 3 (ZP3) fragments. Following modeling, BSHXF (31.53 g/kg/day) was orally administered for 4 weeks. $\mathrm{CD}^{+} \mathrm{T}$ lymphocytes, Tregs, anti-zona pellucida (anti-ZP) antibodies and cytokines were detected using flow cytometry, enzyme-linked immunosorbent assays, reverse transcription-quantitative polymerase chain reaction and immunohistochemistry. The results revealed that BSHXF exhibited an immunoprotective function and reduced inflammatory cell infiltration and damage to the ovary. BSHXF upregulated the percentage of $\mathrm{CD} 4^{+} \mathrm{CD} 25^{+}$forkhead box $\mathrm{P} 3^{+} \mathrm{T}$ cells in the spleen, effectively inhibiting the activation of $\mathrm{CD}^{+} \mathrm{T}$ lymphocytes. The proliferation of Tregs was increased in serum obtained from mice in the BSHXF group in vitro. Anti-ZP antibodies and interleukin-10 and interferon- $\gamma$ levels were decreased in the serum in the BSHXF-treated group. The present study demonstrated that BSHXF had an
\end{abstract}

Correspondence to: Professor Peijuan Wang, Department of Obstetrics and Gynecology, Jiangsu Province Academy of Traditional Chinese Medicine, 100 Hongshan Road, Nanjing, Jiangsu 210028, P.R. China

E-mail: pjwang1961@hotmail.com

${ }^{*}$ Contributed equally

Key words: experimental autoimmune premature ovarian failure, $\mathrm{Bu}$ Shen Huo Xue formula, protective immunity effect, regulatory $\mathrm{T}$ cells, CD4 ${ }^{+} \mathrm{T}$ lymphocyte immunoprotective effect on POF model mice. Additionally, it indicated that the protective mechanisms of BSHXF may be associated with an increase in Treg cells.

\section{Introduction}

Premature ovarian failure (POF), which is characterized by high gonadotropin and low estrogen levels, has a serious impact on women's reproductive and psychological health. The most common presentation is secondary amenorrhea and the main consequence is infertility. Infertility may be the consequence of follicle depletion or a poor response to gonadotropin, which reduces the effectiveness of ovulation induction programs (1). Women with POF not only have premature aging symptoms but are also subject to a significantly increased risk of osteoporosis and cardiovascular disease (2).

Autoimmune abnormality is one of the main causes of POF. Previous studies by Chernyshov et al (3) and Ban (4) indicated that immunoregulation disorder involving an imbalance between $\mathrm{CD}^{+} \mathrm{T}$ and $\mathrm{CD}^{+} \mathrm{T}$ is a leading cause of POF. The researchers also revealed increased autoantibodies of peripheral blood $\mathrm{CD}^{+} \mathrm{CD} 19^{+} \mathrm{B}$ lymphocytes in early cases and high levels of zona pellucida (ZP) antibodies in patients with POF (5). Another study demonstrated that the levels of regulatory $\mathrm{T}$ cells (Tregs) in patients with POF were significantly lower than those in normal controls (6). Thus, the abnormal regulation of Tregs lead to an autoimmune response that harmed the ovaries, which may be responsible for the pathogenesis of POF.

Women with POF are usually treated with hormone replacement therapy to compensate for the decreased ovarian production of sex steroids. However, long-term use of hormone replacement therapy may increase a female's risk of breast and ovarian cancer, venous thromboembolism and stroke (7). Glucocorticoids are used clinically for immunosuppression to treat autoimmune POF; however, there is no known prospective randomized placebo controlled studies that demonstrating the safety and efficacy of immunosuppressive therapy for POF (8). Therefore, the exploitation of effective drugs and treatments is particularly important for the prevention and therapy of POF. 
Bu Shen Huo Xue formula (BSHXF) is a Chinese herbal formulation. Clinical results revealed that BSHXF restored the functions of the ovary and markedly improved the clinical symptoms of POF (9). Early results indicated that BSHXF may protect the ovaries from autoimmune destruction (10). However, the mechanisms require further investigation.

In the present study, an animal model of autoimmune POF was established using internationally recognized ZP3 fragments (11). The protective immunity effects of BSHXF were investigated on the expression of Treg cells and CD4 ${ }^{+}$ $\mathrm{T}$ lymphocytes in mice, anti-ZP antibodies and associated inflammatory cytokines.

\section{Materials and methods}

Reagents and instruments. The amino acid sequence of the murine ZP3 330-342 peptide used in the present study (NSSSSQFQIHGPR) was synthesized by GL Biochem, Ltd. (Shanghai, China), and the amino acid composition was verified by amino acid analysis. Mycobacterium tuberculosis H37RA was purchased from Difco (BD Biosciences, Franklin Lakes, NJ, USA). Incomplete Freund's adjuvant (IFA) was purchased from Sigma-Aldrich (Merck KGaA, Darmstadt, Germany). Qualitative analysis was performed using a HPLC-MS/MS system consisting of Waters 2695 HPLC instrument and Quattro Premier XE MircoMass triple quadrupole tandem mass spectrometer (Waters Co., Milford, MA, USA). Enzyme-linked immunosorbent assay (ELISA) kits for mouse interleukin-10 (IL-10) (\#BMS614-2, IL-10 Mouse ELISA kit) and interferon- $\gamma$ (IFN- $\gamma$ ) (\#BMS6027, IFN alpha Mouse ELISA kit) were purchased from eBioscience (Thermo Fisher Scientific, Inc., Waltham, MA, USA), EnVisionTM horseradish peroxidase rabbit/mouse reagent (\#K5007) was purchased from Agilent Technologies, Inc. (Santa Clara, CA, USA). 3,3'-Diaminobenzidine (DAB) was purchased from Medchemexpress Co., Ltd. (Monmouth Junction, NJ, USA). Mouse anti-CD3 allophycocyanin (APC) (\#17-0031-82), anti-CD4 fluorescein isothiocyanate (FITC) (\#11-0042-86), anti-CD8 phycoerythrin (PE) (\#85-12-0081-82), anti-CD25 FITC (\#11-0250-42), anti-mouse forkhead box P3 (FoxP3) PE (\#12-4771-82), mouse IgG1 isotype control APC (\#MA5-18093), FITC (\#GM4992) and PE (\#GM4993) antibodies were purchased from eBioscience (Thermo Fisher Scientific, Inc.). Red blood cell lysing buffer (\#c3702) and bovine serum albumin (BSA) were purchased from Beyotime Biotechnology Co. (Shanghai, China). RPMI 1640, new born calf serum (NCS), fetal calf serum (FBS) and N-2-hydroxyethylpiperazine-N-2-ethane sulfonic acid (HEPES) were purchased from Invitrogen (Thermo Fisher Scientific, Inc.). Mouse IL-2 (\#P04351) was purchased from R\&D Systems, Inc. (Minneapolis, MN, USA). Carboxyfluorescein succinimidyl ester (CFSE) Cell Proliferation kit (\#C34554) was purchased from Invitrogen (Thermo Fisher Scientific, Inc.). Reverse transcription-quantitative polymerase chain reaction (RT-qPCR) kits and reverse transcription kits were purchased from MBI Fermentas (Thermo Fisher Scientific, Inc.). PCR primers were synthesized by Jinsirui Biotechnology Co., Ltd. (Nanjing, China).

Preparation of BSHXF. The herbal medicine was produced by Jiangsu Yadong Biological Pharmacy Co., Ltd. (Nanjing,
China). BSHXF was composed of Rehmanniae Radix Preparata, Radices Paeoniae Alba, Angelica sinensis, Ligusticum wallichii, Semen Cuscutae, Herba Epimedii, Rhizoma Anemarrhenae, Golden Cypress and Radix Bupleuri. The proportions of these herbs were 10:10:10:10:10:10:10:10:6, respectively. The herbs $(1 \mathrm{~kg})$ were extracted twice with boiling water, and the combined aqueous extracts were concentrated to yield a crude extract (233 g) under reduced pressure, and stored at $4^{\circ} \mathrm{C}$.

Identification of active ingredients in BSHXF using high-performance liquid chromatography (HPLC). Methanol (Tianjin Concord Science Co., Ltd., Tianjin, China) and Milli-Q Academic ultrapure water system (Waters Corp., Milford, MA, USA). All other reagents were of analytical grade (Tianjin Concord Science Co., Ltd.). HPLC with diode-array detection was used to detect peoniflorin (\#23180-57-6), ferulic acid (\#1135-24-6) and icariin (\#489-32-7; Must Bio-Technology Co., Ltd., Chengdu, China). The samples were separated on an Alltima C18 column ( $5 \mu \mathrm{m}, 250 \times 4.6 \mathrm{~mm}$; Waters Corp.) with a mobile phase that consisted of methanol- $0.1 \%$ phosphoric acid (gradient elution) at $25^{\circ} \mathrm{C}$. The injection volume was $20 \mu \mathrm{l}$ with the flow rate of $1 \mathrm{ml} / \mathrm{min}$. The average recoveries of peoniflorin, ferulic acid and icariin were 96.7,97.5 and 99.6\%, and the relative standard deviations were $1.37,3.13$ and $0.96 \%$, respectively. The linear ranges of peoniflorin, ferulic acid and icariin were 0.159-3.970 $\mu \mathrm{g}$ ( $\mathrm{r}=0.9997), 0.048-1.200 \mu \mathrm{g}$ ( $\mathrm{r}=0.9999), 0.207-5.175 \mu \mathrm{g}(\mathrm{r}=0.9999)$. Therefore, the samples were analyzed by HPLC. The contents of the chief components in BSHXF, peoniflorin, ferulic acid and icariin (derived from Radices Paeoniae Alba, Angelica sinensis and Herba Epimedii) were $0.842,0.032$ and $1.608 \mathrm{mg} / \mathrm{g}$, respectively.

Experimental animals. A total of 70 female C57BL/6 mice, 20 male A/J mice (aged 7-9 weeks; weighing, 16.0-18.0 g) and 10 female Wistar rats (aged 7-9 weeks; weighing, 190.0-210.0 g) were purchased from Academy of Military Medical Sciences (Beijing, China) [certification no. SCXK (JUN) 2007-004]. The mice were fed in the laboratory animal room of the Jiangsu Research Institute of Traditional Chinese Medicine [Nanjing, China; certification no. SYXK (SU) 2011-0017]. The animals were housed in a controlled environment (temperature, $23 \pm 2^{\circ} \mathrm{C}$; relative humidity, $42 \pm 5 \%$ with a 12-h light/dark cycle) and had ad libitum access to drinking water and food. Mice were allowed to be acclimatized to the laboratory environment for at least 1 week prior to commencement of testing. The first generation (F1) of hybrid mice was obtained by mating C57BL/6 and A/J mice. B6AF1 mice were studied at 8 weeks of age. Vaginal smears were normal in the cell smear screening. The present study was ethically approved by the Animal Care Ethics Committee of Southeast University (Nanjing, China).

Model establishment and experimental treatment. A total of 75 female B6AF1 mice were randomly divided into three groups: Control $(n=25)$, model $(n=25)$ and BSHXF-treated $(n=25)$ groups. ZP3 peptides were dissolved in double-distilled water to a concentration of $1 \mathrm{mM}$ and sterilized by ultrafiltration. They were then emulsified in an equal volume of IFA containing M. tuberculosis. POF model mice were established 
by injection subcutaneously (s.c.) in the hind footpad and tail root with $0.1 \mathrm{ml}$ emulsion containing $165 \mu \mathrm{g}$ M. tuberculosis

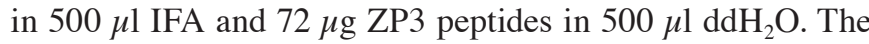
same volume of PBS was injected into a parallel group of mice to represent the Control group. Mice were sacrificed 14 days after immunization in the pre-experiment, which demonstrated POF mouse models were successful. The mice in the BSHXF-treated group were then administered an oral suspension of $31.53 \mathrm{~g} / \mathrm{kg}$ BSHXF once a day for the following 28 days. Mice in the control and model groups were treated with equal amounts of distilled water.

BSHXF-derived serum preparation. A total of $10 \mathrm{Wistar}$ rats were randomly divided into two groups: Blank and BSHXF groups (5 mice per group). At the same time, the mice were orally administrated treatments continuously 2 weeks. BSHXF group rats were administered $18.63 \mathrm{~g} / \mathrm{kg}$ BSHXF whereas the blank group rats were administrated equal volumes of double distilled water. A total of $1 \mathrm{~h}$ after the last BSHXF intragastric administration, serum was acquired from the heart. It was inactivated at $56^{\circ} \mathrm{C}$ for $30 \mathrm{~min}$, filtered through at $0.2-\mathrm{mm}$ sized filter, and stored at $-80^{\circ} \mathrm{C}$ until use.

Estrous cycle staging. Stages of the estrous cycle were determined by examining vaginal cytology. Vaginal washes in saline were transferred to a glass slide, which was air-dried, fixed in methanol for $30 \mathrm{~min}$ at room temperature and stained with Papanicolaou (Hologic, Inc., San Diego, CA, USA) for $10 \mathrm{~min}$ at room temperature. The estrous cycle was staged according to the proportions of leukocytes, nucleated epithelial cells and cornified squamous epithelial cells (12). The sections were observed using light microscopy (Nikon Eclipse 80i; Nikon Corporation, Tokyo, Japan). Images were captured using a digital camera (COOLPIX 950; Nikon Corporation).

Sample collection. The vaginal exfoliate cell smear of mice was observed from day 10 and continued for 4 days. The mice sacrificed on day 14 were examined using hematoxylin and eosin (H\&E) staining in order to determine whether modeling was successful. After 28 days of administration, all remaining mice were sacrificed for analysis. Blood was collected from the mouse orbit. The thymus, spleen, ovary and uterus were extirpated and weighed for the calculation of organ indices. Additionally, the spleens of mice in each group were removed and prepared for flow cytometric analysis, and the left-side ovary and uterus were prepared for H\&E staining for histopathological investigation. The right-side ovary and uterus were immediately frozen to detect $\mathrm{ZP}$ using a direct immunofluorescence assay.

Ovarian and uterus histomorphology. Ovaries were fixed in Bouin's fluid (MXB Biotechnologies, Fuzhou, China; 1:1) fixative for $24 \mathrm{~h}$ at room temperature and embedded in paraffin. Serial sections $(5 \mu \mathrm{m})$ were stained with H\&E for $24 \mathrm{~h}$ at room temperature and examined as coded specimens by an independent observer who was blind to the experimental details. The sections were observed using light microscopy (Nikon Eclipse 80i; Nikon Corporation). Images were captured using a digital camera (COOLPIX 950, Nikon Corporation).
Ovarian immunohistochemistry. The ovaries were isolated from the mice, fixed in $10 \%$ paraformaldehyde for $48 \mathrm{~h}$ at room temperature and paraffin-embedded. Samples were embedded in paraffin wax, treated with xylene and infused into PBS buffer for $4-8 \mathrm{~h}$ in a water bath at $60^{\circ} \mathrm{C}$. Subsequently, samples were rehydrated in a graded alcohol series. Antigen retrieval was accomplished by heating sections to $180^{\circ} \mathrm{C}$ for $10 \mathrm{~min}$ in Citrate buffer (pH 6.2) (Dako; Agilent Technologies, Inc.) and allowed to cool to room temperature. Consecutive 4- $\mu \mathrm{m}$ sections were cut and processed for immunohistochemistry with CD4 (\#RMA-0620), estrogen receptor (ER) (\#kit-0012) and progesterone receptor (PR) (\#kit-0013) at 1:100 concentration (MXB Biotechnologies, Fuzhou, China). Each section was treated with $50 \mu \mathrm{l}$ of primary antibody (CD4/ER/PR) and incubated overnight at $4^{\circ} \mathrm{C}$. Subsequently, each section was treated with $50 \mu \mathrm{l}$ EnVisionTM horseradish peroxidase rabbit/mouse reagent as a secondary antibody overnight at $4^{\circ} \mathrm{C}$. The antibody-binding sites were visualized using DAB for $10 \mathrm{~min}$ at room temperature and the cell nuclei were counterstained with hematoxylin for $10 \mathrm{sec}$ at room temperature. The estrogen/progesterone-receptor-positive clinical breast cancer specimen was used as a positive control and estrogen/progesterone-receptor-negative breast tissue was considered a negative control. The tonsil tissue section served as CD4 positive control and PBS was used in place of the first antibody to serve as a negative control. The positive cells were stained brown. The slides were examined under a light microscope, and representative images were captured from a minimum of 4 or 5 slides from each group. The sections were observed using light microscopy (Nikon Eclipse 80i; Nikon Corporation). Images were captured using a digital camera (COOLPIX 950; Nikon Corporation). All slides were examined in a blinded manner.

Detection of antibodies to ovarian ZP by immunofluorescence. Sections $(5 \mu \mathrm{m})$ of snap-frozen mouse ovaries, which had been fixed in cold acetone for $15 \mathrm{~min}$, incubated by using $3 \%$ bovine serum albumin in PBS for $30 \mathrm{~min}$ at room temperature. Following washing in PBS, slices were dried and treated with $1 \mathrm{ml}$ Alexa Fluor ${ }^{\circledR} 488$ donkey anti-mouse IgG $(\mathrm{H}+\mathrm{L})$ (1:200; \#R37114; Invitrogen; Thermo Fisher Scientific, Inc.) antibody at $37^{\circ} \mathrm{C}$ for $45 \mathrm{~min}$. After washing three times with PBS, slides were viewed and images were captured with an inverted fluorescence microscope (Carl Zeiss AG, Oberkochen, Germany).

Isolation of $\mathrm{CD}^{+} \mathrm{CD}^{25^{+}}$Tregs. $\mathrm{CD} 4^{+} \mathrm{T}$ cells and $\mathrm{CD} 4^{+} \mathrm{CD} 25^{+}$ Tregs were isolated from murine spleen using $\mathrm{CD}^{+}{ }^{+} \mathrm{T}$ cell isolation and $\mathrm{CD} 4^{+} \mathrm{CD} 25^{+}$Treg isolation kits (Thermo Fisher Scientific, Inc., Waltham, MA, USA), respectively, following the manufacturer's protocols.

Flow cytometry (FCM) analysis. To assess the changes in splenic Treg cells in each group of mice, the levels of $\mathrm{CD}^{+}$ $\mathrm{CD}^{2} 5^{+}$FoxP $^{+} \mathrm{T}$ cells were measured using FCM. Spleens of mice were harvested, passed through a $70-\mu \mathrm{m}$ cell strainer, centrifuged at $300 \mathrm{x} \mathrm{g}$ for $5 \mathrm{~min}$ at $4^{\circ} \mathrm{C}$ and resuspended in a red blood cell lysing buffer at $37^{\circ} \mathrm{C}$ for $2 \mathrm{~min}$ to lyse the red blood cells, whereby erythrocytes from the spleen were lysed in ammonium chloride buffer and the remaining living cells 
washed and resuspended in PBS. A single-cell suspension was prepared. Two sets of experiments were performed. The first set used a total of $1 \times 10^{6}$ cells stained with the following conjugated antibodies: CD3-APC, CD4-FITC and CD8-PE. The second set used a total of $1 \times 10^{6}$ cells stained with CD4-FITC, CD25-APC and FoxP3-PE. FoxP3 and cytokine staining were performed according to the manufacturer's intracellular staining protocol, and appropriate isotype antibodies were used as controls.

In vitro CFSE proliferation assay. The culture media consisted of RPMI 1640 supplemented with 2\% NCS, 1\% HEPES, $1 \%$ anti-CD3/28 mAbs and 1\% FBS. Culture media was freshly composed before usage and stored in a refrigerator at $4^{\circ} \mathrm{C}$. Isolated $\mathrm{CD}^{+}{ }^{+} \mathrm{CD} 25^{+}$Treg cells were seeded into 96 well plate, each well containing $1 \times 10^{5}$ Tregs. The cells were labeled with CFSE (Invitrogen; Thermo Fisher Scientific, Inc.), stimulated with IL-2 (40 U/well) and BSHXF-derived serum/PBS (10 $\mu \mathrm{l} /$ well). T cells were cultured in media at $37^{\circ} \mathrm{C}$ under $5 \%$ $\mathrm{CO}_{2}$ saturated humidity for 2 days. After $24 \mathrm{~h}$, the media were replaced with fresh media. A total of $48 \mathrm{~h}$ later, the cells were harvested and analyzed using flow cytometry. Detection of $\mathrm{CD} 4{ }^{+} \mathrm{CD} 25^{+}$Treg proliferation using a CFSE kit (\#C34554; Invitrogen, Thermo Fisher Scientific, Inc.) following the manufacturer's protocols.

Determination of cytokine levels in mouse serum. The blood of each mouse was centrifuged at $1,000 \mathrm{x} \mathrm{g}$ for $10 \mathrm{~min}$ at $4^{\circ} \mathrm{C}$ to obtain serum for cytokine ELISA. ELISA kits were used to detect changes in the levels of IFN- $\gamma$ and IL-10 cytokines in the mice. The aforementioned ELISA kits were used for quantitative determination of the aforementioned cytokines in the serum samples of mice. Cytokine concentrations were determined using the relevant standard curves.

Expression levels of FoxP3 mRNA. Total RNA was isolated from $\sim 30 \mathrm{mg}$ of spleen tissue using TRIzol (Invitrogen; Thermo Fisher Scientific, Inc.) according to the manufacturer's instructions. The RNA concentration was quantified with a Spectrophotometer (Eppendorf, Hamburg, Germany). RNA was reversed transcribed into cDNA using reverse transcriptase (Takara Shuzo Co., Tokyo, Japan) at $37^{\circ} \mathrm{C}$ for $1 \mathrm{~h}$. A total of $500 \mathrm{ng}$ of RNA was reverse transcribed in a $20-\mu 1$ system using a Revert Aid First-Strand cDNA synthesis kit (Fermentas; Thermo Fisher Scientific, Inc.) and the following thermocycling conditions were used: $25^{\circ} \mathrm{C}$ for $10 \mathrm{~min}, 50^{\circ} \mathrm{C}$ for $15 \mathrm{~min}, 85^{\circ} \mathrm{C}$ for $5 \mathrm{~min}$, followed by a holding temperature of $4^{\circ} \mathrm{C}$. Subsequently, $1 \mu \mathrm{l}$ complementary DNA was used as the template for qPCR using SYBR-Green PCR master mix (with ROX; Invitrogen; Thermo Fisher Scientific, Inc.) and a 7300 Real-Time PCR system (Applied Biosystems; Thermo Fisher Scientific, Inc.). Each qPCR reaction contained $0.1 \mathrm{mM}$ each primer, $5 \mu \mathrm{l}$ SYBR-Green PCR master mix including AmpliTaq Gold DNA polymerase with buffer, dNTP mix, SYBR-Green I and ROX reference dyes and $10 \mathrm{mM} \mathrm{MgCl}$, and $1 \mu 1$ template cDNA in a $10-\mu 1$ total reaction volume. The typical amplification program included activation of the enzyme at $95^{\circ} \mathrm{C}$ for $10 \mathrm{~min}$, followed by 45 cycles of denaturation at $95^{\circ} \mathrm{C}$ for $15 \mathrm{sec}$, then annealing and extension at $60^{\circ} \mathrm{C}$ for $30 \mathrm{sec}$. The $\mathrm{Cq}$ (cycle threshold) value for each gene was determined using the automated threshold analysis function of the PCR instrument. The relative expression levels of FoxP3 against GAPDH were calculated using the $2^{-\Delta \Delta \mathrm{Cq}}$ method (13). The primer sequences for PCR designed using Primer Premier 5.0 software (Premier Biosoft International, Palo Alto, CA, USA) on the basis of GenBank sequences, were as follows: FoxP3 forward, 5'-CTGGACAACCCAGCCATGAT-3' and reverse, 5'-ACATTGATCCCAGGTGGCAG-3'; GAPDH forward, 5'-CTGAAAATCAATAGCACGAAC-3' and reverse, 5'-ATGGAGCCACCGATCCACA-3'.

Statistical analysis. Each experiment was performed as least thrice, and the data are presented as the mean \pm standard error where applicable. All statistical comparisons were analyzed by means of one-way analysis of variance followed by Tukey's test using SPSS 21.0 software (IBM Corp., Armonk, NY, USA). $\mathrm{P}<0.05$ was considered to indicate a statistically significant difference.

\section{Results}

General status of mice following model establishment. Symptoms of the model group, including a flaccid tail, staggering gait and irritability, appeared sequentially in experimental mice from day 8 . The ovaries of mice in the model group were smaller than those of controls and the uterine volume was also reduced.

Examination of the vaginal smears in the control group (Fig. 1A) demonstrated that the mice had a regular estrous cycle whereas the model group presented irregular, prolonged and even arrested estrous cycles. Regular sex cycles were identified and the ovulation of mice was increased in the BSHXF-treated group compared with the model group.

The results of ovarian H\&E staining demonstrated that compared with the control group, there were more primordial follicles, and less typical growing and mature follicles in the cortex of mouse ovaries in the model group, and a large amount of apoptosis was observed in all these follicles (Fig. 1B).

\section{Changes of indices following administration of BSHXF}

Body weight. The body weight of mice in each group decreased markedly at $>2$ weeks following model establishment. However, as the time was extended the body weight gradually increased. Compared with the model group, the body weight of the BSHXF-treated group increased more rapidly, but the most rapid increase was observed in the control group. Notably, BSHXF may have a protective effect on autoimmunity damage in mice with POF (Fig. 2A).

Organ indices. The spleen index in the model group mice was increased but the thymus, uterus and ovary indices were decreased compared with those in the control group $(\mathrm{P}<0.05)$. Compared with the model group, the ovarian and thymus indices in the BSHXF-treated group mice increased significantly $(\mathrm{P}<0.01)$, whereas the spleen index decreased and uterus index increased to a non-statistically significant extent (P>0.05; Fig. 2B).

Pathological changes on the ovary and uterus. The results of ovarian $\mathrm{H} \& \mathrm{E}$ staining revealed that the number of 


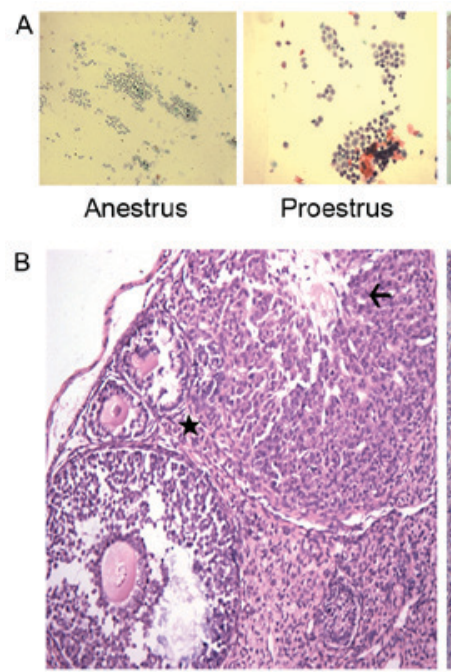

Control group
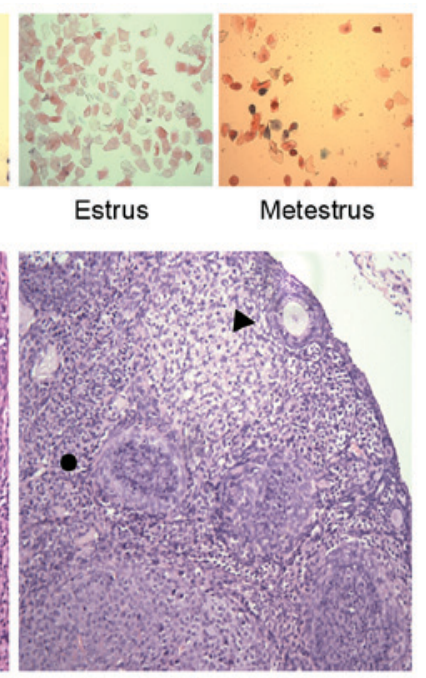

Model group
Figure 1. (A) Normal mouse vaginal smears (magnification, x100; pap staining; yellow filter). (B) Ovarian pathology (magnification, x200; hematoxylin and eosin staining) prior to and following modeling compared with the control group. The images revealed that there were primordial follicles, and fewer typical growing and mature follicles in the cortex of mouse ovaries in the model group compared with the control group, and a large number of apoptotic cells were observed in these follicles. Primary follicles are indicated by a star; corpus luteum is indicated by an arrow; primordial follicle is indicated by a triangle; and a dysplastic follicle is indicated by the circle.

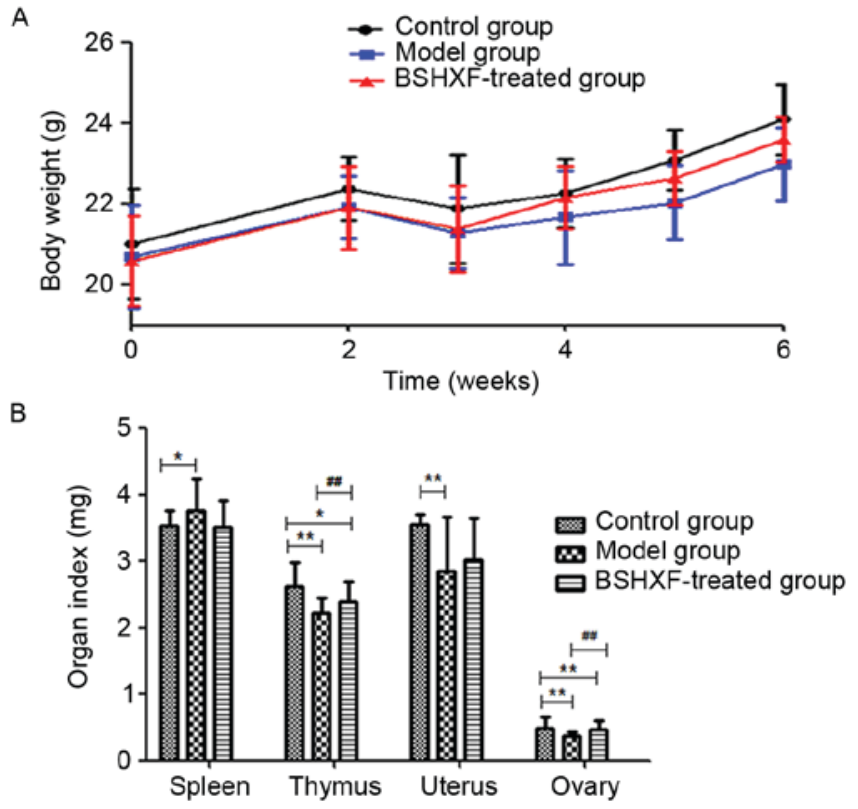

Figure 2. (A) Changes in mouse body weights prior to and following BSHXF administration. Prior to modeling, the weights of the mice in the control, model and BSHXF-treated groups were 21.024 $\pm 1.353,20.700 \pm 1.287$ and $20.588 \pm 1.113(\mathrm{P}>0.05)$. Following modeling, the body weights were markedly reduced in the model group compared with the control. At the end of the 6-week experimental period, the body weights were $24.108 \pm 0.861$, $23.612 \pm 0.564$ and $22.984 \pm 0.903$ for the control, model and BSHXF-treated groups, respectively, and the body weight in the model group was significantly decreased compared with that in the control and BSHXF-treated groups $(\mathrm{P}<0.01)$. (B) Changes of organ indices prior to and following BSHXF administration. The index values in the control, model and BSHXF-treated groups were: Thymus index: $2.619 \pm 0.371,2.220 \pm 0.235$ and $2.400 \pm 0.305 \mathrm{mg}$; spleen index: $3.543 \pm 0.227,3.767 \pm 0.476$ and $3.520 \pm 0.409 \mathrm{mg}$; uterus index: $3.561 \pm 0.149,2.854 \pm 0.825$ and $3.018 \pm 0.640 \mathrm{mg}$; ovary index: $0.498 \pm 0.164$, $0.382 \pm 0.043$ and $0.465 \pm 0.137 \mathrm{mg}$. ${ }^{*} \mathrm{P}<0.05$ and ${ }^{* *} \mathrm{P}<0.01$ vs. the control group; ${ }^{\# \#} \mathrm{P}<0.01$ vs. the model group. BSHXF, Bu Shen Huo Xue formula.
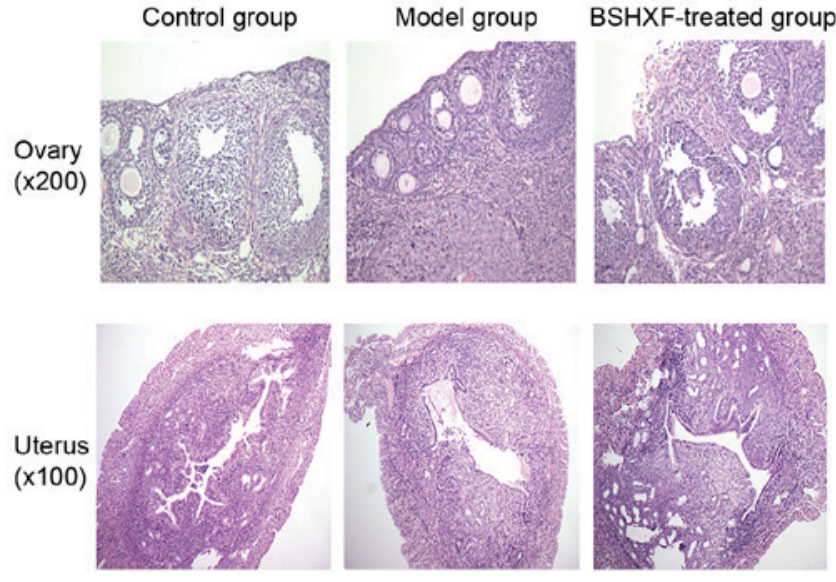

Figure 3. Observation of pathological changes in hematoxylin and eosin stained ovary and uterus sections of mice under a light microscope. BSHXF, Bu Shen Huo Xue formula.

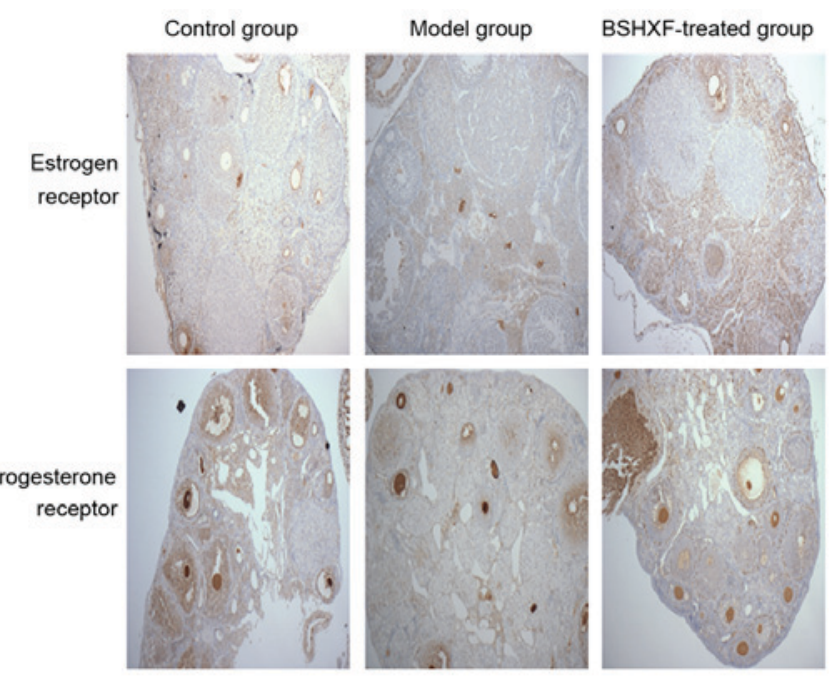

Figure 4. Expression changes of estrogen and progesterone receptors in the ovaries (magnification, x100). BSHXF, Bu Shen Huo Xue formula.

follicles, particularly developing follicles, was greater in the BSHXF-treated group compared with the model group, and the lymphocytes were less involved in the follicle with some lymphocyte infiltration in the interstitium. The results of $\mathrm{H} \& \mathrm{E}$ staining of the uterus demonstrated that the uterine endometrium in the model group was thinner than that in the control group, secretory material was decreased in the epithelium, the basic layer of the uterine gland was decreased, the gland cavity decreased and reticulations of the inner membrane were also reduced. However, the endometrium was thickened in the BSHXF-treated group. It is noteworthy that BSHXF induced changes in endometrial structure, and endometrial hyperplasia and thickening (Fig. 3).

\section{Immunohistochemical detection of changes in $E R$ and $P R$} expression in the ovaries. Immunohistochemical studies indicated that the expression of the ER and PR in the ovary in the model group was decreased relative to that in the control group. After 4 weeks of continuous administration, the expression of ER and PR in the ovary increased. Estrogen and progesterone 
A

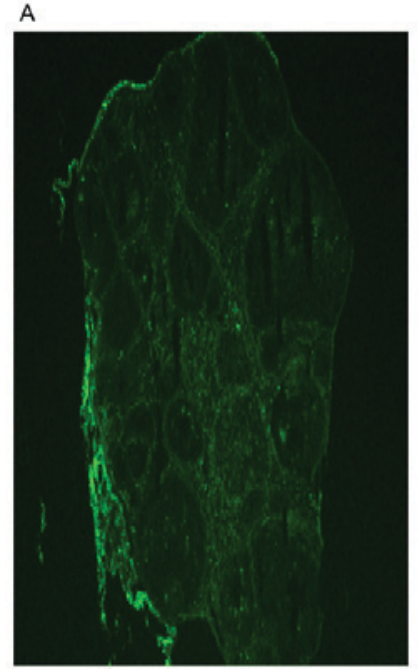

Control group
B

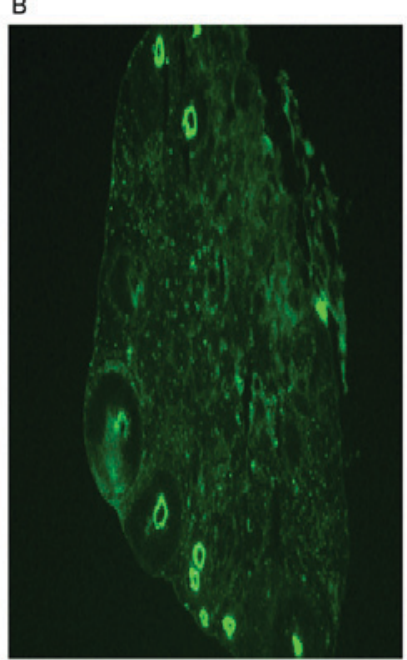

Model group

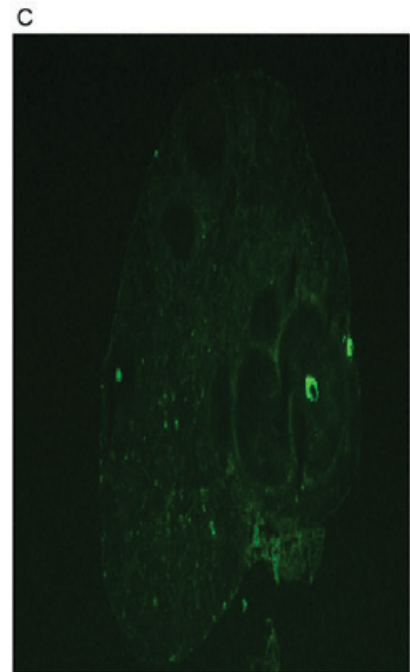

BSHXF-treated group

Figure 5. Immunofluorescence examination of ovarian sections to detect antibodies to zona pellucida (magnification, $\mathrm{x} 40$ ). (A) Control group, (B) model grop and (C) BSHXF-treated group. BSHXF, Bu Shen Huo Xue formula.

exert their functions by combining with their corresponding receptors. It appears that the expression of ER and PR in mice ovary may be promoted by BSHXF (Fig. 4).

Immunofluorescence detection of ovarian ZP. No strong immunofluorescence was exhibited in the control group whereas bright green immunofluorescence was observed in the model group. In the BSHXF-treated group, a few spots of immunofluorescence were observed, and the expression of ZP antibody was clearly decreased compared with that in the control group (Fig. 5).

Regulation of $\mathrm{CD}^{+} \mathrm{T}$ lymphocyte immune response. Immunohistochemical results for $\mathrm{CD}^{+}$are depicted in Fig. 6A. The model mice exhibited increased lymphocyte infiltration around the follicle compared with the control mice. Lymphocyte infiltration was clearly reduced following treatment with BSHXF.

Proliferation of $T$ cells. FBS or medicated serum was added to CFSE-labeled $\mathrm{CD} 4^{+} \mathrm{CD} 25^{+}$Treg cells following separation, and the cells were then cultured. After 2 days, the effect on cell proliferation was detected by FCM. This revealed greater proliferation in the medicated serum group compared with the control group (Fig. 6B) indicating that BSHXF in the serum was able to induce mouse $\mathrm{CD}^{+}{ }^{+} \mathrm{CD} 25^{+}$Treg cell proliferation.

ELISA analysis of inflammation-related cytokines in the serum. The levels of IL-10 and IFN- $\gamma$ in the serum of model mice was significantly increased compared with that in the control group mice. However, the levels of these cytokines were significantly decreased in BSHXF-treated mice compared with the model mice $(\mathrm{P}<0.001$; Fig. 6C).

FCM analysis of splenic $C D 4^{+} C D 25^{+} F_{0 x P 3^{+}} T$ cells. The results of the FCM analysis are presented in Fig. 7A. A significant reduction in $\mathrm{CD}^{+}{ }^{+} \mathrm{CD} 25^{+} \mathrm{FoxP}^{+} \mathrm{T}$ cells in the model mice compared with the control group was observed $(\mathrm{P}<0.01)$, and treatment with BSHXF significantly increased the number of these cells compared with the model group $(\mathrm{P}<0.05)$.

RT-qPCR analysis of FoxP3 mRNA expression in the spleen. As demonstrated in Fig. 7B, FoxP3 mRNA in the spleen of model mice was significantly decreased compared with that of the control group mice $(\mathrm{P}<0.001)$. Treatment with BSHXF significantly increased FoxP3 mRNA levels in the spleen compared with those in the model and control groups $(\mathrm{P}<0.001)$.

\section{Discussion}

Autoimmunity may be closely associated with the pathogenesis of POF. A previous study indicated that human autoimmune oophoritis is a pathological entity encountered in 5\% of women with POF (14). Autoimmune oophoritis is the main mechanism of ovarian immune damage. ZP antigen immunization results in increasing atretic follicles and disorders of the local immune milieu in the ovaries, which facilitates depletion of follicles in different phases and leads to POF. The induction of autoimmune oophoritis in B6AF1 mice using ZP3 has been described in the literature $(11,15,16)$. Therefore, this model with the same dosage was selected for use in the present study. The results indicate that the estrous cycle was dysfunctional in the model mice, with a lower ovarian and uterine viscera coefficient and an atrophied ovary and endometrium. Ovarian pathology revealed inflammatory cell expression, which generated more atretic follicles. Ovarian follicles were infiltrated by inflammatory cells and anti-ZP antibody was expressed. Additionally, immunohistochemical results for the ovaries revealed that ER and ER expression was downregulated. Furthermore, ovarian follicles were surrounded by a large number of infiltrating $\mathrm{CD}^{+} \mathrm{T}$ lymphocytes. These indicated that the model of autoimmune POF was successfully established.

$\mathrm{CD}^{+} \mathrm{T}$ lymphocytes are the main cells involved in cellular immunity, and their activation can cause immune damage $(3,4)$. Additionally, $\mathrm{CD} 4{ }^{+} \mathrm{CD} 25^{+}$Tregs belong to a T-lymphocyte 


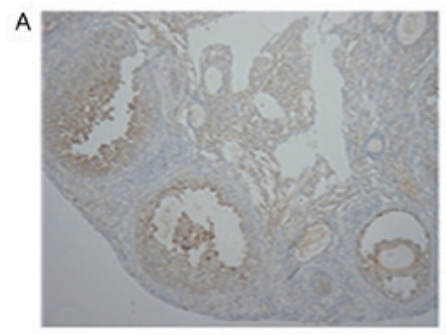

Control group

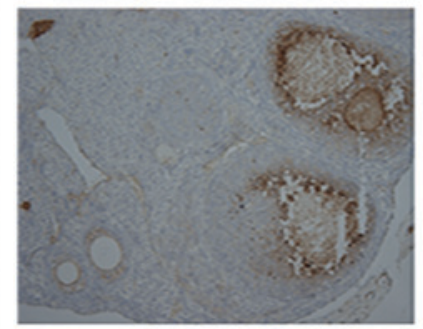

Model group

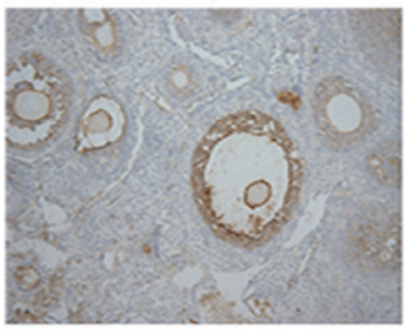

BSHXF-treated group

B
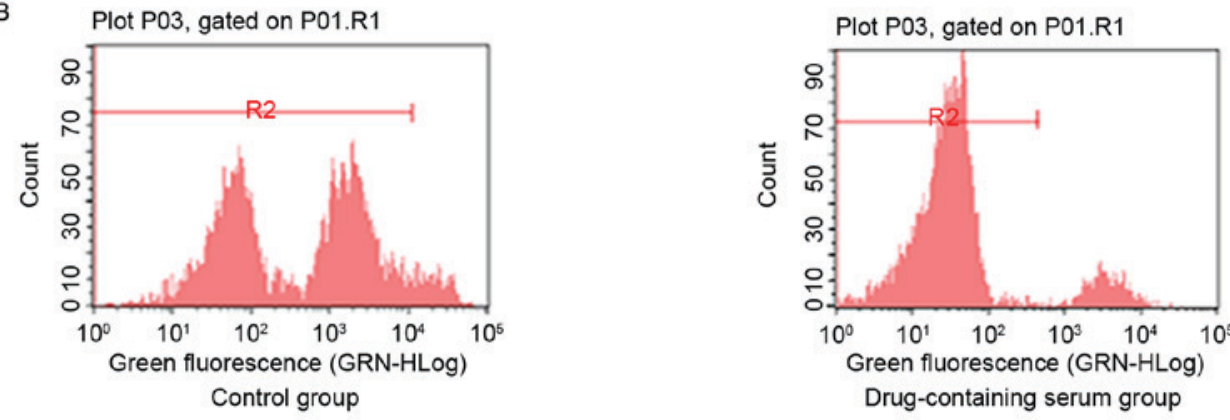

C
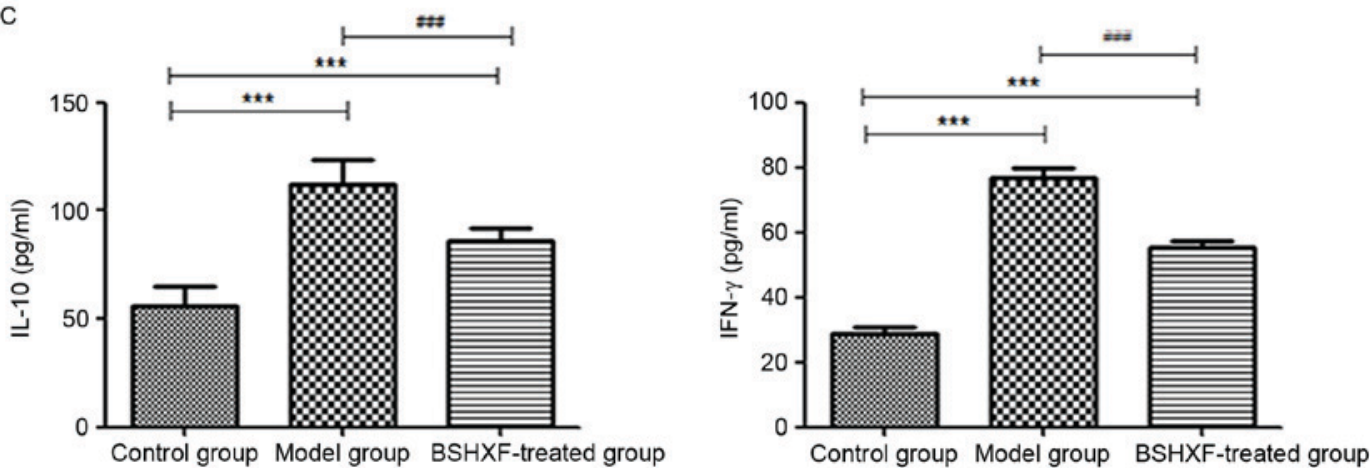

Figure 6. (A) Immunohistochemical staining of $\mathrm{CD}^{+}$in the ovaries (magnification, $\mathrm{x} 200$ ). (B) CFSE-based proliferative assay for CD4 ${ }^{+} \mathrm{CD} 25^{+}$Treg lymphocytes. (C) ELISA analysis of IL-10 and IFN- $\gamma$ expression in the spleen of the three groups. In the control, model and BSHXF-treated groups, the levels of IL-10 were $55.647 \pm 9.185,111.918 \pm 11.282$ and $85.742 \pm 6.166$, respectively, and the levels of IFN- $\gamma$ were $28.831 \pm 11.024,76.844 \pm 13.736$ and $55.183 \pm 11.595$, respectively. ${ }^{* * *} \mathrm{P}<0.001$ vs. the control group; ${ }^{\# \#} \mathrm{P}<0.001$ vs. the model group. BSHXF, Bu Shen Huo Xue formula; IL, interleukin; IFN- $\gamma$, interferon- $\gamma$.
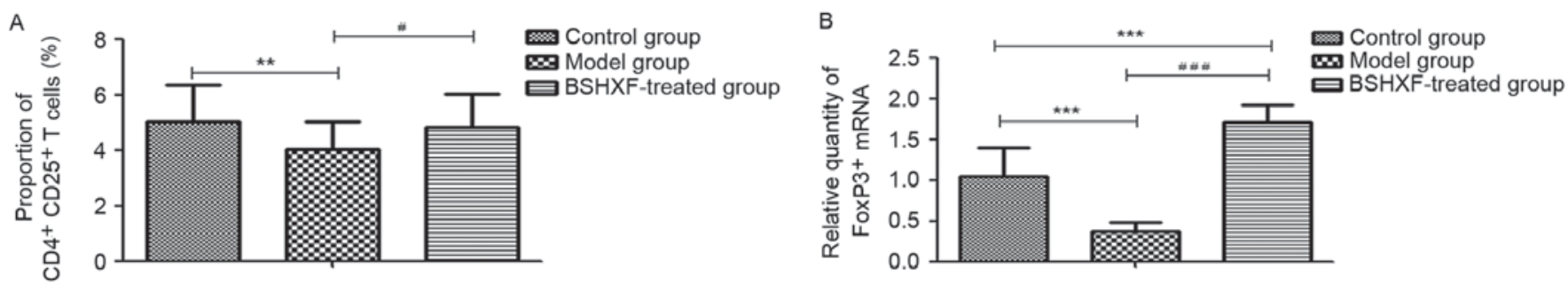

Figure 7. (A) Effect of BSHXF on the expression of $\mathrm{CD}^{+} \mathrm{CD} 25^{+}$FoxP3 ${ }^{+} \mathrm{T}$ cells in the spleen of mice. The average Treg proportions in the control, model and BSHXF-treated groups were $5.033 \pm 1.312,4.034 \pm 0.975$ and $4.810 \pm 1.222 \%$, respectively. Following administration of BSHXF, the Treg level was higher than that of the model groups. (B) Effect of BSHXF on the expression of FoxP $3^{+} \mathrm{T}$ cell mRNA in the spleen of mice. The FoxP3 ${ }^{+} \mathrm{mRNA}$ level in the spleen in model mice was $0.373 \pm 0.108$, which was significantly decreased compared with that in the control group mice $(\mathrm{P}<0.001)$. Furthermore, the FoxP $3^{+} \mathrm{mRNA}$ expression level in the spleen in the BSHXF-treated group was $1.704 \pm 0.222$, which was significantly increased compared with that in the control and model groups $(\mathrm{P}<0.001) .{ }^{* *} \mathrm{P}<0.01$ and ${ }^{* * *} \mathrm{P}<0.01$ vs. the control group; ${ }^{\#} \mathrm{P}<0.05$ and ${ }^{\# \# \#} \mathrm{P}<0.001$ vs. the model group. BSHXF, Bu Shen Huo Xue formula; Treg, regulatory T cells; FoxP3, forkhead box P3.

subset with an immunity regulation function. They have an important function in the maintenance of a stable internal environment and self-immune tolerance. Furthermore, the reduction or dysfunction of these cells may be correlated with autoimmune diseases $(17,18)$. A previous study demonstrated that Tregs are able to prevent and even reverse autoimmune disease progression in mice (19). FoxP3, which is closely associated with the development and function of Tregs, is essential for Treg cell differentiation and the functioning of transcription factors (20). In the present study, FCM methods demonstrated 
that $\mathrm{CD} 4^{+} \mathrm{CD} 25^{+}$Treg cells were decreased in model mice. Compared with the control group mice, FoxP $3^{+}$mRNA levels in the spleen were significantly decreased in model mice. The results of the present study imply that autoimmune POF may be associated with $\mathrm{T}$ lymphocyte levels, and be particularly closely correlated with Treg levels.

Inflammation is important in the progression of autoimmune POF (21). IL-10 significantly inhibits the synthesis of mononuclear cells and the release of inflammatory mediators (22). Furthermore, IFN- $\gamma$, a potent immunomodulatory cytokine, is a member of the IFN family that regulates immune responses through the activation of mononuclear macrophages (23). The results of the present study demonstrate that serum levels of IL-10 and IFN- $\gamma$ increased in the model mice. The data of the present study indicated that increases in inflammatory and anti-inflammatory factors increased with the occurrence of the inflammatory response. Similar results have been observed for other autoimmune diseases (24).

Experimental studies have revealed that Bu Shen Huo Xue inhibits the specific immune damage of residual follicles in the ovaries, thereby restoring ovarian function in mice with POF, with additional preventive effects on the incidence of POF $(25,26)$. Earlier studies have demonstrated that traditional Chinese medicine is able to attenuate immune injury. Angelica Sinensis, Rehmanniae Radix Preparata, Radix Paeoniae Alba and Ligusticum Wallichii in the $\mathrm{Bu}$ Shen Huo Xue formulation are the main components of Siwutang. Pharmacological experiments have revealed that Siwutang enhances immune function, improves the spleen and the thymus index of aging mice, and regulates multiple functional states of the immune system (27). Additionally, an Angelica Sinensis extract is able to increase the number of immune cells and regulate immune functions (28). The peoniflorin in Radix Paeoniae Alba and ligustrazine and ferulic acid in Ligusticum Wallichii have been demonstrated to promote the transformation and proliferation of lymphocytes in mice $(29,30)$. Furthermore, Semen Cuscutae and Herba Epimedii have immunoregulatory functions $(31,32)$. These findings indicate that numerous compounds present in BSHXF contribute to immune function.

The results of the present study demonstrated that BSHXF elevated the ovarian index, increased the number of mature follicles, reduced the levels of anti-ZP antibodies, promoted the expression of ER and PR and improved ovarian functions. In addition, it decreased the spleen index in mice with autoimmune POF (although not significantly), and effectively inhibited the activation of $\mathrm{CD}^{+} \mathrm{T}$ lymphocytes, increased Treg cells and promoted the mRNA expression of FoxP3. Serum of mice treated with BSHXF was used to cultivate $\mathrm{CD} 4{ }^{+} \mathrm{CD} 25^{+}$Treg cells separated from the spleen, and the proliferation of the cells was increased. Therefore, it is considered that BSHXF may improve the immune function and status of autoimmune POF mice by increasing Treg cells.

The present study provides a basis for further research into the efficacy of BSHXF in the treatment of POF. However, the BSHXF formulation is complex and the components exert multi-targeted effects. Thus, other mechanisms by which BSHXF influences disease in POF mice remain poorly understood and require further investigation.
In the present study, the mechanisms of autoimmune POF are indicated to be associated with a reduction in the number of $\mathrm{CD} 4^{+} \mathrm{CD} 25^{+}$Treg cells and increased activation of $\mathrm{CD} 4{ }^{+}$ $\mathrm{T}$ lymphocytes, leading to an excessive immune response of the ovaries. BSHXF may exert significant protective immunity on $\mathrm{POF}$ in mice by increasing the number of $\mathrm{CD} 4{ }^{+} \mathrm{CD} 25^{+}$Treg cells to inhibit the activation of $\mathrm{CD} 4^{+} \mathrm{T}$ lymphocytes.

\section{Acknowledgements}

The present study was supported by the National Natural Science Foundation of China (grant no. 81303136), Jiangsu Province Natural Science Foundation of China (grant no. SBK20151606) and Jiangsu Province Bureau of Traditional Chinese Medicine (grant no. LZ13088). The authors would like to thank Professor Jian Zhang, Professor Peng Cao and Professor Meng Cao for their invaluable help in the processing of the experiment.

\section{References}

1. Beall SA and DeCherney A: History and challenges surrounding ovarian stimulation in the treatment of infertility. Fertil Steril 97: 795-801, 2016.

2. Wu X, Cai H, Kallianpur A, Li H, Yang G, Gao J, Xiang YB, Ji BT, Yu-Tang, Zheng W and Shu XO: Impact of premature ovarian failure on mortality and morbidity among Chinese women. PLoS One 9: e89597, 2014.

3. Chernyshov VP, Radysh TV, Gura IV, Tatarchuk TP and Khominskaya ZB: Immune disorders in women with premature ovarian failure in initial period. Am J Reprod Immunol 46: 220-225, 2001.

4. Ban H: Peripheral blood cell immune detection and its clinical significance of premature ovarian failure patients. China Modern Med 17: 84-85, 2010.

5. Shi X, Shu Q and Zhang J: Detection and analysis of autoimmunity data on patients of premature ovarian failure. Prog Obstet Gvnecol 16: 591-593, 2007 (In Chinese).

6. Xie J, Dong Y, Liang Z and He W: Changes and significances of $\mathrm{CD} 4^{+} \mathrm{CD} 25^{+} \mathrm{T}$ regulatory cells in patients with premature ovary failure. Reprod Contracep 33: 224-227, 2013.

7. Farquhar C, Marjoribanks J, Lethaby A, Suckling JA and Lamberts Q: Long term hormone therapy for perimenopausal and postmenopausal women. Cochrane Database Syst Rev: CD004143, 2009.

8. Kalantaridou SN, Braddock DT, Patronas NJ and Nelson LM: Treatment of autoimmune premature ovarian failure. Hum Reprod 14: 1777-1782, 1999.

9. Yu N: Traditional Chinese medicine treatment of premature ovarian failure in clinical research. Hunan J Tradit Chin Med 24: 94-96, 2006.

10. Tang CL, Li F, Sun L and Li DJ: Therapeutic Effect of Bushen Huoxue recipe on autoimmune premature ovarian failure mice established by immunization with recombinant porcine zona pellucida 4 antigen. Chin J Integr Med 19: 439-445, 2003.

11. Fu L, Zhao Y and Li S: The establishment of the animal model of premature ovarian failure. J Reprod Med 15: 179-183, 2006 (In Chinese).

12. Cora MC, Kooistra L and Travlos G: Vaginal cytology of the laboratory rat and mouse: Review and criteria for the staging of the estrous cycle using stained vaginal smears. Toxicol Pathol 43: 776-793, 2015.

13. Livak KJ and Schmittgen TD: Analysis of relative gene expression data using real-time quantitative PCR and the 2(-Delta Delta C(T)) method. Methods 25: 402-408, 2001.

14. La Marca A, Brozzetti A, Sighinolfi G, Marzotti S, Volpe A and Falorni A: Primary ovarian insufficiency: Autoimmune causes. Curr Opin Obstet Gynecol 22: 277-282, 2010.

15. Rhim SH, Millar SE, Robey F, Luo AM, Lou YH, Yule T, Allen P, Dean J and Tung KS: Autoimmune disease of the ovary induced by a ZP3 peptide from the mouse zona pellucida. J Clin Invest 89: 28-35, 1992. 
16. Kimura S, Matsumoto T, Matsuyama R, Shiina H, Sato T, Takeyama $\mathrm{K}$ and Kato S: Androgen receptor function in folliculogenesis and its clinical implication in premature ovarian failure. Trends Endocrinol Metab 18: 183-189, 2007.

17. Belkaid Y: Regulatory T cells and infection: A dangerous necessity. Nat Rev Immunol 7: 875-888, 2007.

18. Mottet C, Uhlig HH and Powrie F: Cutting edge: Cure of colitis by $\mathrm{CD} 4{ }^{+} \mathrm{CD} 25^{+}$regulatory T cells. J Immunol 170: 3939-3943, 2003.

19. Vojdani A and Erde J: Regulatory T cells, a potent immunoregulatory target for CAM researchers: Modulating tumor immunity, autoimmunity and alloreactive immunity (III) Evid Based Complement Alternat Med 3: 309-316, 2006.

20. Shevach EM: Mechanisms of foxp $3^{+} \mathrm{T}$ regulatory cell-mediated suppression. Immunity 30: 636-645, 2009.

21. Said RS, El-Demerdash E, Nada AS and Kamal MM: Resveratrol inhibits inflammatory signaling implicated in ionizing radiation-induced premature ovarian failure through antagonistic crosstalk between silencing information regulator 1 (SIRT1) and poly(ADP-ribose) polymerase 1 (PARP-1) Biochem Pharmacol 103: 140-150, 2016.

22. Polz-Dacewicz M, Strycharz-Dudziak M, Dworzański J, Stec A and Kocot J: Salivary and serum IL-10, TNF- $\alpha$, TGF- $\beta$, VEGF levels in oropharyngeal squamous cell carcinoma and correlation with HPV and EBV infections. Infect Agent Cancer 11: 45, 2016.

23. Chen $\mathrm{J}$ and Ivashkiv LB: IFN- $\gamma$ abrogates endotoxin tolerance by facilitating Toll-like receptor-induced chromatin remodeling. Proc Natl Acad Sci USA 107: 19438-19443, 2010.

24. Nie X, Deng R, Xiang L, Jiang P and Xue Q: Reno-protective effect and mechanism study of Huang Lian Jie Du Decoction on lupus nephritis MRL/lpr mice. BMC Complement Altern Med 16: 448, 2016.
25. Cai LR, Li DJ and Sun XX: Experimental study on preventive and therapeutic effect of Bushen Huoxue recipe on autoimmune premature ovarian failure model. Zhongguo Zhong Xi Yi Jie He Za Zhi 21: 126-129, 2001 (In Chinese).

26. Dong L, Jiang L, Men W and Zhu NS: Preventive and therapeutic effects of Bushen Huoxue Recipe on autoimmune premature ovarian failure in mice. Zhong Xi Yi Jie He Xue Bao 6: 294-297, 2008 (In Chinese).

27. Liang H, Zhu M, Sun Y, Wang X and Xu J: The immunoregulatory research progress of Sijunzi decoction and siwu decoction. Inf Traditional Chin Med 29: 136-137, 2012 (In Chinese).

28. Liu SP, Dong WG, Wu DF, Luo HS and Yu JP: Protective effect of angelica sinensis polysaccharide on experimental immunological colon injury in rats. World J Gastroenterol 9: 2786-2790,2003.

29. Xu Y and Dong Y: Radix paeoniae alba total glycosides on mice lymphocytes in vitro value-added and active research. Chin Arch Traditional Chin Med 31: 914-917, 2013.

30. Zhang T,Zhao R,Liu Z, Shang Y, Wang M and Wang X: Ligustrazine effects on T lymphocyte activation value. Chin J Gerontol 29: 1658-1659, 2009 (In Chinese).

31. Zhou P: Epimedium immune pharmacological research progress. Chin J Traditional Med Sci Technol 17: 279-280, 2010.

32. Lin HB, Lin JQ, Lin Jian-Qun, Lu N and Yi XY: Comparative study on immune enhancement effects of four kinds of dodder seeds in Shandong province. Zhong Xi Yi Jie He Xue Bao 1: 51-53, 2003 (In Chinese).

This work is licensed under a Creative Commons

Attribution-NonCommercial-NoDerivatives 4.0

International (CC BY-NC-ND 4.0) License. 